\title{
POSTGRADUATE STUDENTS' UNDERSTANDING OF MIXED METHODS RESEARCH DESIGN AT THE PROPOSAL STAGE
}

\author{
T. E. Mabila \\ Department of Research Administration and Development \\ University of Limpopo \\ Polokwane, South Africa \\ e-mail: thembinkosi.mabila@ul.ac.za
}

\section{ABSTRACT}

Mixed methods research (MMR) design by postgraduate students planning their research projects is on the rise. However, little is known about their conception of this relatively new methodology as their research begins. We report on an exploratory inquiry into the understanding of MMR by postgraduate students in a South African comprehensive university as revealed in their research proposals. We employed document analysis to examine a total of 67 proposals, and found conflicting and divergent interpretations of MMR. Most proposals gave no philosophical foundation for choosing particular methodological combinations; many were unclear about the selected design, lacked pertinent methodological information, provided insufficient justification for choice of study design, and lacked rigour in addressing data collection and analysis. This situation may have detrimental consequences for the university's growth of research capacity in this area and future research culture. We conclude with MMR recommendations for research committees, supervisors and candidates for research degrees.

Keywords: mixed methods, research proposal, postgraduate students, comprehensive university, research culture

\section{INTRODUCTION}

Scholarly interest in postgraduate students' conceptions of research methods has recently started gathering momentum (Kawulich, Garner and Wagner 2009). Despite this promising scenario, a remarkable fact, observed by authors including Vermunt (2005), Murtonen and Lehtine (2005), Meyer, Shanahanb and Laugkschc (2005), and more recently by Stubba, Pyhältöb and Lonkaac (2014) is the scarcity of literature relating to student conceptions of research methodology. Where available, in cases such as Sandelowski (2004) and Davies and Hughes (2014), it has focused on young and emerging researchers' employment of qualitative and quantitative approaches as independent paradigms. Mixed methods research (MMR), which combines the two, is relatively new and has been described as 'a growing area of methodological choice for many academics and researchers from across a variety of discipline areas' (Cameron 2011, 96). It is therefore particularly helpful for developmental supervision 
purposes to examine the level of understanding that students have of this kind of research as early as at the proposal stage of their master's and doctoral degree, and to ascertain areas of methodological strength as well as challenges to be addressed during the research project to follow.

Tashakkori and Teddlie $(2010,803)$ point to the fact that MMR has expanded and acquired acceptance in that:

... it has gone through a relatively rapid growth spurt ... it has acquired a formal methodology that did not exist before and is subscribed to by an emerging community of practitioners and methodologists across the disciplines. In the process of developing a distinct identity, as compared with other major research communities of researchers in the social and human sciences, mixed methods has been adopted as the de facto third alternative, or third methodological movement.

Despite this development, and the fact that successful implementation of MMR depends on a researcher's knowledge and personal attitudes towards the methodology (Starr 2012), the conceptualisation of this design among students at postgraduate level has received little scholarly attention. Where available, studies have looked into perceptions of postgraduate students regarding the use of MMR in thesis and dissertation research (Ukwuoma 2015). These include, for example, the investigation by Ponce and Pagán-Maldonado (2015) of MMR in education; Stockman's (2015) exploration of the dynamics involved in pursuing and achieving a doctorate through MMR; and Guetterman's (2016) study of 'What distinguishes a novice from an expert mixed methods researcher'. Others such as Ukwuoma (2015) and MunyaradziMuchacha and Mtetwa (2015) have reported on barriers militating against postgraduate students' use of MMR. To the best of our knowledge, the only available published study on students' actual understanding of MMR is by Plowright (2013), who reported on the extent to which his sample of postgraduate students in the United Kingdom understood the principles of mixed methods in educational research. His study findings revealed that there is some confusion and inconsistencies in the views of postgraduates about foundational methodological and philosophical principles associated with the execution of MMR.

We could find no studies that have focused on how MMR is understood by postgraduate students in general nor, more specifically, within the context of a South African comprehensive university that is still developing its research culture. The country's higher education system accommodates different emphases through its three different kinds of universities: traditional universities that provide theoretically orientated university degrees, universities of technology that focus on vocationally orientated education, and comprehensive universities that offer a combination of academic and vocational diplomas and degrees (CHET 2010). According to 
Gibbon (2004, 5), a comprehensive university is characterised by:

Diversity, through the offering of a diverse range of academic programmes (vocational, careerfocused, professional and general formative) of both university and technikon type. Accessibility, through the opportunities created by a variety of entry and exit points. Student mobility, through developing strong vertical and horizontal articulation pathways. Responsiveness, through the development of a suite of educational programmes and research foci appropriate to local, regional and national needs. Flexibility, through the strengthening of relationships with community, civic, government, business, and industry partners for local and regional development. Flexibility should characterise the institution's ability to meet the human resource needs of the local (and wider) context through its training programmes, and to contribute to the development of the communities it serves through the application and extension of its knowledge and expertise.

Gibbon's description reveals the broad range of academic offerings in a comprehensive university that creates challenges in developing a research culture, in generating quality research, and in training future generations of researchers adequately. Hence, it is important to assess the quality of research methodology understanding in general at such universities, and of MMR in particular, given its relative novelty and its increasing popularity and acceptance as an approach of choice.

The present study focused on postgraduate students' understanding of MMR at the early proposal stage of their research degree, as this is the point at which the foundation is laid for their entire project. The research questions that guided our study were: To what extent do postgraduate students at the beginning of their master's or doctoral research studies understand MMR? What strengths and challenges are in evidence at the proposal stage? The next section provides the motivation and background to our study.

\section{BACKGROUND}

This study grew out of Creswell's (2011) foundational work in MMR, which provides a clarion call for the design and evaluation of MMR studies. Various authors (notably Burrows 2013; Plowright 2013) have provided interpretations of how MMR design should be understood. For Creswell (2013), MMR design and evaluation are typically characterised by six core features: the collection of both qualitative and quantitative data, the analysis of both qualitative and quantitative data, persuasive and rigorous procedures for both sets of data, the integration of these two data sources, the use of a specific MMR design that involves a concurrent or sequential integration with equal or unequal emphases, and an approach to research that has a sound philosophical foundation. Understanding these criteria is vital for MMR of appropriate quality, and therefore crucial for research and teaching in institutions of higher learning, and especially for enabling satisfactory postgraduate outcomes. 
Plowright's (2013) study, which examined postgraduate students' understanding of the principles of MMR, was informed by questionnaire and interview responses, and his sample comprised students in the field of educational research. Our study covered a broader multidisciplinary terrain and its focus was on the crucial research proposal stage of a postgraduate student's master's or doctoral study. As Wong $(2016,1)$ avers, 'one’s research is only as good as one's proposal. An ill-conceived proposal dooms the project even if it somehow gets through the thesis supervisory committee.' It is essential for postgraduate students entering their studies - as well as for any researchers who wish to produce quality research reports - to possess clear understanding of their methodology from the very onset of their studies.

When providing a nurturing or supportive research environment, such understanding is also important for those directly involved in early postgraduate development. These include the supervisor, who is responsible for guiding the postgraduate student, and the research committee, which is responsible for evaluating the research proposal and offering constructive feedback. By understanding thoroughly the prescripts of MMR design, supervisors can support postgraduate students effectively as they conceptualise their methodology design, and they can assess progress with confidence and provide productive input. When the proposal is submitted for formal approval, the research committee can scrutinise, assess and provide effective reportback to the supervisor and postgraduate student during the evaluation process. This process is structured to yield quality proposals, which should, in turn, allow the smoothest possible completion of successful postgraduate studies as well as desired levels of throughput.

The decision to situate the present study in a comprehensive university goes to the heart of South Africa's broader national goals and expectations for upliftment, equity, and growth through the outcomes of post-apartheid higher education. In this context, the country's comprehensive universities find themselves in a terrain where research development has become too urgent to ignore. South Africa currently produces $28 \mathrm{PhDs}$ per million of the population; this 'is low by international standards', according to the National Development Plan 2030 (NPC 2012, 278), which made 'a number of bold proposals for universities and doctorate studies in particular'. Amongst other issues, it prioritises:

- increasing the number of master's and PhD students: by 2030, over 25 per cent of university enrolments should be at postgraduate level;

- producing more than 100 doctoral graduates per million of the country's population by the year 2030 . 
This means that not only do research intensive universities need to promote and support postgraduate study, but comprehensive universities, too, have a crucial role to play in achieving these ambitious objectives.

Most comprehensive universities draw students from predominantly rural environments, who, even more than 20 years after the end of apartheid, are still largely characterised by low socio-economic status and a history of poor access to well resourced educational facilities. Upliftment is therefore a key aspect of the work of these institutions. For this reason, and because of the relatively recent growth of MMR across disciplinary areas, this approach to research and the criteria for evaluating it need to be well understood and implemented by comprehensive, and even research intensive universities, in order to produce quality research proposals and studies.

When the supervisor and the research committee possess and apply clear understanding of the methodology and assessment, support for postgraduate researchers in preparing and approving MMR postgraduate studies becomes effective from the foundation level of their studies onwards. The suggested interactions are illustrated in Figure 1, which presents the process for postgraduate support at this stage. Understanding MMR allows supervisors to scrutinise, assess, and give accurate feedback to students; master's and doctoral students to prepare and submit quality proposals; and members of the Research Committee to offer constructive suggestions at the approval stage of a proposal.

The next section briefly summarises the history and philosophy of MMR and situates this study in the available literature.

\section{MIXED METHODS RESEARCH}

Mixed methods research developed into prominence at the turn of the millennium. Its pioneers include research methodologists and scholars from a variety of disciplines, notably Abbas Tashakkori, Anthony Onwuegbuzie, Charles Teddlie, Gert Biesta, John Creswell, Kathleen Collins, Martyn Descombe and Vicki Clark. The MMR approach has been employed with real success in various disciplines, including business studies and management (Cameron and Molina-Azorin 2011); sociology, psychology and education (Mertens 2014); health care sciences (Munhall 2012); and human resources and marketing (Harrison and Reilly 2011).

As a movement, MMR has 'developed its own philosophy, theoretical, methodological, analytical as well as practical foundations and constructs' (Cameron 2011, 96). Creswell (2013), Clark and Creswell (2011), Biesta (2010), Denscombe (2008), Tashakkori and Teddlie (2010a; 2010b), and Onwuegbuzie (2002) all agree that it is pragmatic in nature. Biesta (2010, 101), for example, points out that 'pragmatism replaces the underlying assumption of western 


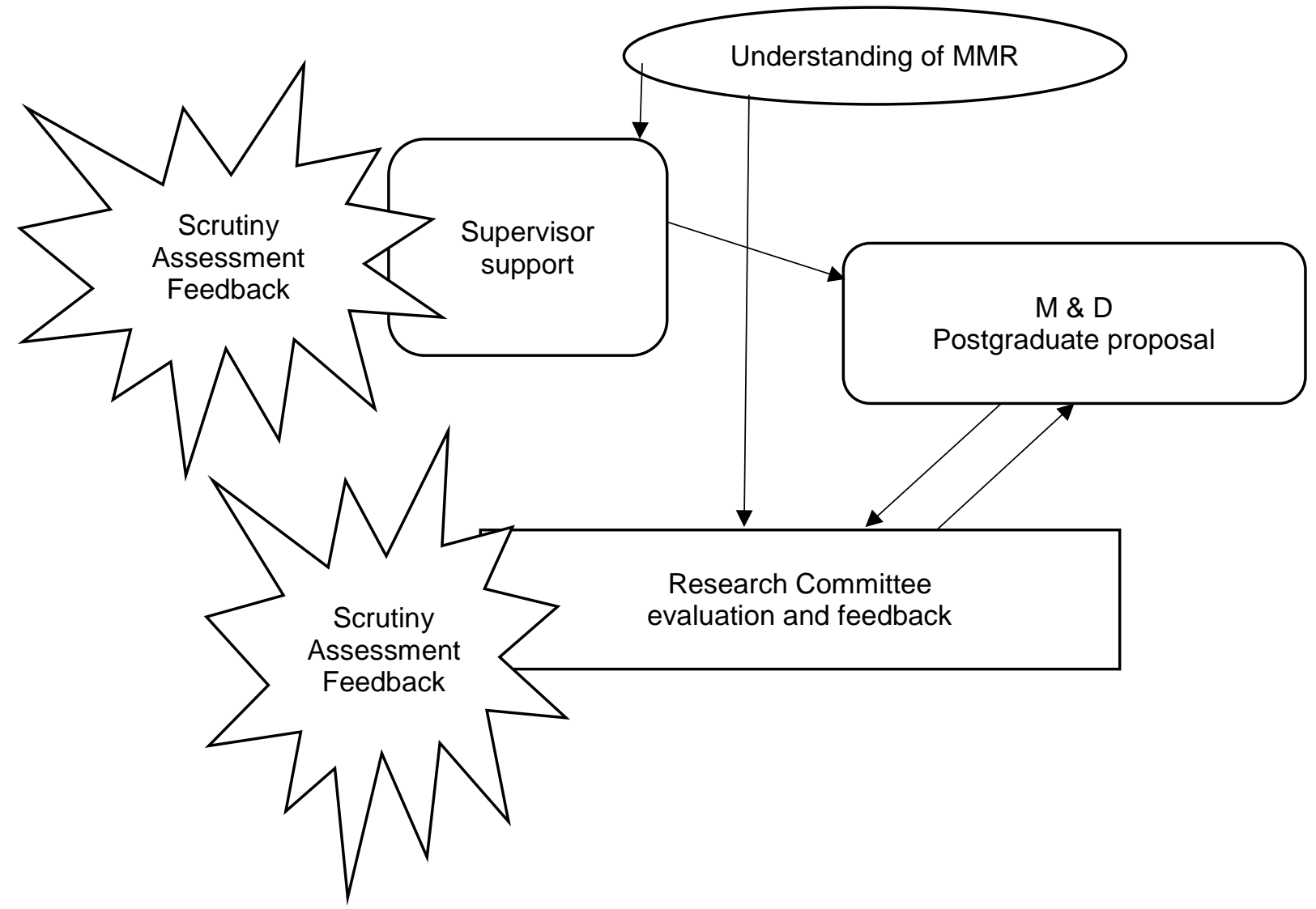

Figure 1: A process for support of postgraduate MMR proposal preparation.

epistemology as founded by Descartes and Hume, which assumes that reality consists of two totally different substances, mind and matter'. Hence, the MMR philosophical underpinnings are attributed to Dewey's stance, which does not assume the 'mind-world' scheme, but understands nature as a moving whole of interacting parts. The philosophical situation of this movement, according to Biesta (2010) as well as Tashakkori and Teddlie (2010b), can be summed up as follows: interactions occur in a natural environment; experience results from transactions of living organisms and their environments; hence all forms of experience are considered real. Knowledge, therefore is concerned with the conditions and consequences of experience and not limited to physical phenomena.

In the light of these developments, our focus was to explore postgraduate understanding of MMR, and how this understanding provides the basis for supporting research students adequately in the design and execution of studies following MMR methodology. 


\section{METHODOLOGY}

\section{Context}

The present study was conducted at a South African comprehensive university that is in the process of growing its research culture. MacGregor $(2010,1)$ explains that 'comprehensive universities include former research-intensive institutions whose performance in terms of research, success rates, postgraduates and staff qualifications declined following mergers with historically disadvantaged institutions, as well as the three formerly disadvantaged universities'. The institution where the study was conducted is one of these formerly disadvantaged universities and has four faculties: Humanities; Management Sciences and Law; Health Science; and Sciences and Agriculture. Most of the postgraduate students come from the surrounding community, which is mainly rural and characterised by households that can be classified as having low socio-economic status. The academic staff are mainly early career researchers (ECRs), who, typically, have obtained a doctoral or other research postgraduate qualification only within the last five years (Bazeley 2003). Most staff members are themselves still engaged in postgraduate studies whilst also teaching and supervising postgraduate research projects. Hence, many of the supervisors have limited supervision skills and are 'overloaded' with work and supervision responsibilities. Aptly described, the context for the study is characterised by disadvantage, in contrast with South Africa's well resourced urban researchintensive universities, which, as MacGregor (2010) indicates, at present produce the bulk of the country's postgraduates and future academics, are generally well endowed, and have high student success and graduation rates, high proportions of academic staff with PhDs, low staffstudent ratios, and consequently high research outputs.

The importance of the population selected for the present study is the national need to rapidly increase the number of doctoral graduates and the concomitant need to grow a quality academic workforce in the face of rising calls for greater access to quality education by movements such as '\#feesmustfall'. In essence, it is worth noting that building the capacity of early career researchers and postgraduates is about institutions as much as it is about individuals (Harle 2012), especially in view of growing pressure to improve research profiles and culture at all South African universities.

\section{Sources of data}

The main source of data for our study was the set of agendas for the University's Research Ethics Committee for the 2015 academic year. Following a purposive approach to sampling, all 
research proposals appearing in the five agenda documents for the academic year were included. Out of the total of 248 submissions, 44 master's and 23 doctoral proposals followed an MMR methodology and were extracted as sources of data.

\section{Design}

Within the framework of concurrent triangulation in MMR (Creswell 2013), the present study adopted a retrospective cohort design, which uses information collected in the past and kept in files or databases (Mann 2003). We conducted a census of all 2015 research proposals submitted to the Research Ethics Committee (REC) for ethical clearance, including all postgraduate master's and doctoral proposals. All those that claimed to apply MMR were filed for evaluation. Other studies that adopted a single approach in design or that were submitted for non-degree purposes were discarded.

\section{Data collection}

Several authors have recommended criteria for evaluating MMR design, most notably Long et al. (2002), Leech et al. (2010), Bamberger (2012), and Burrows (2013). Burrows (2013) provides a model that underwent a rigorous multi-phase analysis to create a rubric for use in MMR evaluation. In the present study, all the proposals that adopted MMR design were evaluated using a checklist that we adapted from Burrows's Rubric for Evaluating Mixed Methods (BREMM) research, consisting of 14 standards (or criteria) through which the philosophical, theoretical, methodological, analytical and practical foundations, and constructs of MMR designs can be assessed. For our purpose, 12 out of the 14 standards were deemed applicable to the evaluation of MMR proposals; the two that were omitted deal with the evaluation of completed MMR studies. Table 1 summarises the checklist used in the present study.

Table 1: Checklist for evaluating mixed methods research design proposals, adapted from Burrows (2013)

\begin{tabular}{|l|l|l|l|}
\hline Question & Evaluation standard & & \\
\hline Q1 & The study's purpose statement is clearly stated. & Yes & No \\
\hline Q2 & The research design is clearly stated. & Yes & No \\
\hline Q3 & $\begin{array}{l}\text { The methods of how the study will be conducted (data collection } \\
\text { and analysis) are clearly stated. }\end{array}$ & Yes & No \\
\hline Q4 & $\begin{array}{l}\text { An explanation of how data will be mixed/ integrated is clearly } \\
\text { stated. }\end{array}$ & Yes & No \\
\hline Q5 & $\begin{array}{l}\text { The research question(s)/objectives to guide the study are clearly } \\
\text { stated. }\end{array}$ & Yes & No \\
\hline Q6 & The reason for mixing in the study is clearly stated. & Yes & No \\
\hline Q7 & There is a clearly stated explanation of what 'value will be added' by & Yes & No \\
\hline
\end{tabular}




\begin{tabular}{|l|l|l|l|}
\hline Question & Evaluation standard & & \\
\hline Q8 & mixing/integrating the data. & & \\
\hline Q9 & The researcher's philosophical paradigms are clearly stated. & Yes & No \\
\hline Q10 & $\begin{array}{l}\text { The review of literature pertaining to mixed methods research is } \\
\text { clearly stated. }\end{array}$ & Yes & No \\
\hline Q11 & How the methodology fits the purpose of the study is clearly stated. & Yes & No \\
\hline Q12 & The phase in which the mixing/ integration occurs is clearly stated. & Yes & No \\
\hline
\end{tabular}

In addition, we also qualitatively evaluated the proposals, commenting about issues pertaining to MMR design in each. This qualitative evaluation formed the basis of the thematic analysis that followed.

\section{Data analysis}

The checklist data were subjected to analysis using descriptive statistics and presented through frequency charts. A thematic approach advocated by Braun and Clarke (2006) was adopted to analyse the qualitative data. The evaluations of the research proposals provided rich and thick descriptions of emergent issues.

\section{Quality criteria}

For a quality MMR study, Healy and Perry (2000) and Golafshani (2003) advise that it is essential to understand and blend the necessary relevant criteria. Since our study followed a concurrent triangulation design, which adopts the equal use of qualitative and quantitative approaches, quality assurance (Onwuegbuzie, Johnson and Collins 2011) was achieved through the application of measures drawn from both paradigms. For the quantitative part, reliability and validity were assured, while for the qualitative component, trustworthiness and dependability issues were addressed.

\section{Reliability}

Consistency during the data collection process brings reliability (Johnson and Christensen 2008). Hence, the proposal evaluators were extensively trained in the design of MMR prior to engaging in the evaluation task. Their training involved discussions and agreements for the adaptation and adoption of the checklist rubric used for data collection. After the initial evaluation exercise, each case was discussed by all four evaluators in the study before the evaluations were confirmed. 


\section{Validity}

Triangulation is often mentioned as a central method of 'validating' research (Ritchie, Lewis, Nicholls and Ormston 2003). In this study, validation was achieved by ensuring that each evaluation of a proposal was verified by any two other members of the research team. McMillan and Schumacher (2014) call this action corroboration, and indicate that it can be brought about by collecting data through a variety of sources. In the case of our study, the data were collected using the checklist as well as qualitative evaluation.

\section{Trustworthiness}

To safeguard reliability in qualitative research, trustworthiness is critical (Seale 1999). Thus the data we collected through the checklist and evaluation of the proposal documents were recorded with honesty, and safely stored for perusal and verification by any interested party following the proper channels of accessing data.

\section{Dependability}

Closely related to trustworthiness is dependability (Tashakkori and Teddlie 2008). For dependability in the study, we ensured that responses were obtained by posing the same questions adapted from Burrows (2013) to all evaluations. Prior to this, the instrument for data collection was piloted to make sure that it pursued relevant information without unintended ambiguity.

\section{RESULTS AND DISCUSSION}

Our checklist was employed to identify the presence or absence of the postgraduates' conceptual knowledge and skills relating to MMR design. This tool was used by four researchers in the project, to evaluate the 67 purposefully selected proposals employing an MMR design.

\section{Faculty distribution of MMR proposals}

Analysis of the 67 proposals revealed that MMR was used across all four faculties in the university. This finding is in line with Cameron (2011, 96), who observed that 'mixed methods research is a growing area of methodological choice for many academics and researchers from across a variety of discipline areas'. In our study, however, most proposed MMR studies were from the Faculty of Humanities (31; 46\%) and included research projects in education, languages and the social sciences; next came those from the Faculty of Health Sciences (19; 
$28 \%$ ) in the areas of health care and medicine; a close third came from the Faculty of Management and Law (15; 22\%); and just a couple from Science and Agriculture (2; 3\%).

In the health sciences disciplines, the use of MMR has been described as a quiet revolution (O’Cathain 2009). This observation was supported in the profile of our data, with proposals from the health sciences emerging as the second most popular area for the use of MMR studies.

\section{Checklist evaluation}

Analysis of responses to the checklist, which set the criteria for evaluating the MMR proposals, revealed that, while some aspects of MMR were reasonably well understood, most were inadequately presented. The postgraduate students' understanding of MMR design in our study fell into three major categories: aspects that, on the whole, were well understood; those that were reasonably well understood; and those that were poorly understood.

Figure 2 provides a summary of the checklist analysis. It shows that the postgraduate proposals revealed differing postgraduate students' understanding of aspects of MMR. Our discussion of the understanding of aspects of MMR design is therefore presented as follows: Category A (that is, Q1, Q2 and Q3) comprises areas showing good student understanding of aspects of MMR; Category B (Q4, Q6, Q9, Q10 and Q11) comprises areas showing fair student understanding; and Category C (Q5, Q7, Q8 and Q12) comprises areas showing poor understanding.

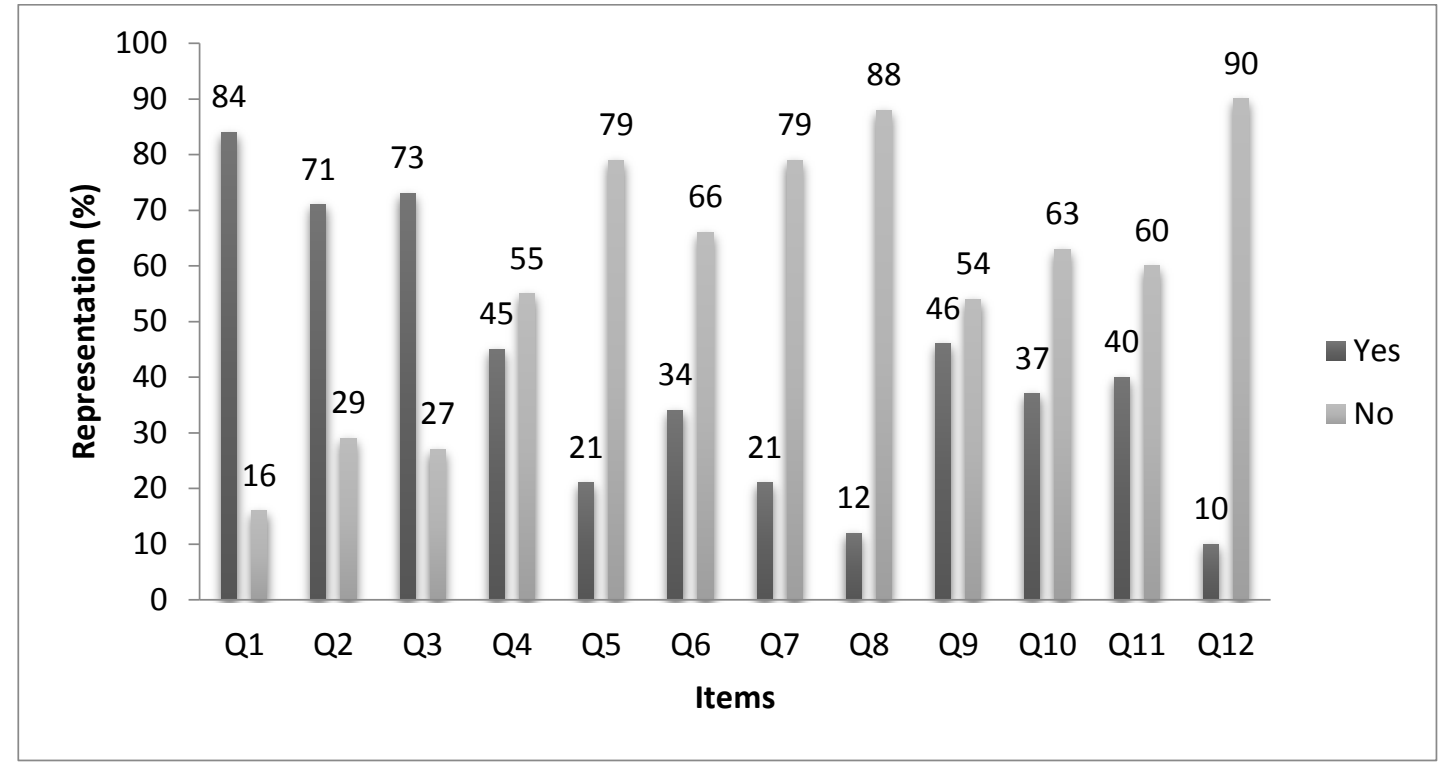

Figure 2: Percentage representation of the understanding of aspects of mixed methods research.

In general, the proposals indicated good understanding of certain fundamental aspects of MMR design. The proposed study's purpose (Q1) and research design (Q2) were in most cases clearly 
stated (84\% and $71 \%$, respectively). In addition, about three-quarters of the proposals (73\%) clearly stated their methods of data collection and analysis (Q3).

However, less than half of the proposals explained clearly how data would be integrated (Q4); gave reasons for mixing (Q6); offered a literature review relating to MMR design (Q9); provided explanations of what value would be added by mixing data (Q10); or indicated the phase in which the mixing was to occur (Q11) in the proposed study.

Of greatest concern was the fact that very few (less than a quarter) of the proposals had clearly stated research questions (Q5); showed the value to be added by mixing or integrating data (Q7); presented relevant philosophical paradigms (Q8); or addressed the replicability of the study (Q12).

The ability to provide a study's purpose clearly is essential in the execution of any research design, including MMR. Bazeley (2004) and Hanson (2006), for instance, stipulate that study objectives define the specific aims of a MMR study and should be clearly stated in the introduction of the research protocol. Bazeley explains that, when the study purpose is made clear, its focus becomes a superordinate goal that limits tensions in the mixing of methods, and Odom et al. (2005) maintain that research question(s) and objectives are amongst important quality indicators and guidelines for evidence of effective practices in implementing MMR. Designing and developing an appropriate and relevant MMR study can be difficult (Hanson 2006). Hence, the ability by most of the students in our sample to clearly state the research design in the MMR proposals that we evaluated was viewed as a strong positive. Moreover, Easton, McComish and Greenberg (2000) point to the fact that when researchers provide clearly stated methods of data collection and analysis, they are better able to avoid common pitfalls such as the omission of pertinent data and misinterpretation of collected data. Broad understanding of the data collection and analysis aspect of MMR design in our postgraduate proposals was also viewed as encouraging.

Following Mertens (2014), MMR study proposals need to explain how the two types of data will be integrated. Hence, Bulsara (1997) advises that researchers should clarify the phase at which integration of data occurs, and be obliged to provide reasons for mixing. For Creswell (2013), these details also help to ensure that designs are fit for purpose by matching research design to research questions and by mixing methods in a way that obtains the most valuable data possible. Hence, according to Leech and Onwuegbuzie (2009), integration details should always be supported by the provision of relevant MMR literature, particularly at the primary level of the study. According to O'Cathain, Murphy and Nicholl (2010), integration of the qualitative and quantitative components is an important and essential aspect of MMR, and they 
observe that recent empirical studies using this approach frequently lack explanations of integration. For this reason, it was cause for concern that less than half of our postgraduate students’ proposals showed sufficiently clear understanding of this issue.

Creswell (2013) advises that explanations of what value will be added by applying MMR and mixing the data in proposed studies (Hurmerinta-Peltomäki and Nummela 2006) should be backed by appropriate philosophical assumptions. Most of the proposals that we analysed reflected poor coverage of this important aspect of the approach. Cameron (2011), for instance, argues that such philosophical assumptions guide the direction of the collection and analysis of data as well as the mix of qualitative and quantitative data in a single study. In view of this stance, it was a matter of concern that a large majority of the research proposals in our study did not convey awareness of this important consideration. In addition, we were not surprised to find that most of the proposed studies were found not to be replicable, since replicating in MMR studies is considered a difficult exercise and not likely to become a popular venture even amongst experienced researchers (Mertens 2014).

\section{Overall assessment of the MMR proposals}

The responses to the evaluation criteria were grouped and scored: 'Yes' responses received a positive score, 'No' responses received a negative one. The best possible evaluation score for a proposal was 12. All proposal scores were computed, then delineated as follows: proposals scoring 6 and above indicated adequate understanding of MMR; those scoring below 6 indicated inadequate understanding of MMR.

We found that 55 per cent of our sample demonstrated inadequate understanding and 49 per cent revealed adequate understanding of mixed methods. The lowest score from all proposals was -3 while the highest was 9 . When the mean of the evaluation scores was considered, it was established that, in general, the students scored an average of about 6.4. We concluded that this cohort had a borderline understanding of MMR design. Our results were then considered together with the findings from observations made during the qualitative document evaluations undertaken in our study.

The notes and comments made by the evaluators as they assessed the proposals were subjected to a thematic analysis as recommended by Braun and Clarke (2006), and yielded four themes: issues about philosophical assumptions; reasons for the choice of MMR; issues relating to the design; and aspects of data collection and analysis. The next section briefly discusses the findings from the thematic analysis. 


\section{Thematic analysis}

First, consideration by the evaluators of issues pertaining to philosophical foundations in the MMR proposals that were scrutinised revealed that, in most cases, no philosophical foundations were provided. This finding confirmed the results revealed by the analysis of Question 8 of the checklist indicating lack of clarity about philosophical foundations.

Second, analysis of the evaluators' comments revealed that most of the selected proposals made no attempt to clarify what prompted the selection of a MMR design. We found this to be a serious weakness in the design of the proposed studies, especially when considering Creswell's $(2013,216)$ warning of 'limitations to consider when choosing this approach'; inadequate motivation for the use of the chosen design would imply that the studies would not be sufficiently tightly crafted to ensure quality of execution.

Third, the evaluators had concerns that students did not clarify whether the study would involve concurrent or sequential integration of data collection and analysis, nor did they provide information as to whether the selected design would involve equal or unequal employment of qualitative and quantitative approaches, as advocated for MMR by Teddlie and Tashakkori (2011).

Finally, most of the proposals omitted key aspects of data collection and analysis. The evaluators remarked that even when proposals mentioned that data collection and analysis would involve both qualitative and quantitative means, inadequacies included (a) lack of clarity regarding how rigour would be ensured; and (b) problems with the use of terminology specific to qualitative/quantitative research approaches. Creswell (2014) makes it clear that, in MMR design, issues of rigour need to be balanced between the qualitative and the quantitative strands. Equally, depending on the balance of the two strands, it is important to justify the approach in terms of quality assurance. This means that if the elements of quality come mainly from one strand of research methodology rather than the other, it becomes important to provide justification, since lack of rigour can negatively affect a study's acceptability. Our analysis revealed that the postgraduate students' proposals often neglected this factor. With regard to use of terminology, our analysis also revealed that the students had problems with the classification of specific research concepts and use of correct technical terms. There was prominent misuse and confusion of terms such as pragmatism, validity, reliability, credibility, trustworthiness, and triangulation, as well as, to some extent, incorrect nomenclature relating to data collection tools such as questionnaires and interview schedules. Hence, we point out that students need to know that opting for MMR is not a permit to generalise nomenclature traditionally associated with one or other paradigm. 


\section{CONCLUDING REMARKS}

The issues raised in this study offer a useful and practical contribution to existing knowledge about the level of genuine understanding of MMR design among postgraduate students within a range of disciplines at the planning stage of their study, specifically in the context of a South African comprehensive university in the process of developing its research culture. We illustrate the application of a set of criteria designed for standard use in the support and evaluation of MMR research projects at the early proposal stage. This method can be applied more widely to assess understanding of an MMR design to be applied, and to identify areas of weakness that need to be addressed. Our approach, therefore, is relevant not only in a comprehensive university setting but also in other contexts - that is, wherever it is necessary to evaluate and improve MMR research proposals.

The growth and evolution of MMR brings new challenges as research committees, supervisors, and students engage with its novelty and do their best to apply best practice in guiding their own and their students' research projects. We conclude, therefore, that, the broader effort to grow a research culture - especially in the comprehensive university context - requires a developmental model that takes into account the actual knowledge needs of all three groups involved: the supervisor who is the primary leader in a postgraduate research study; the research committee that scrutinizes, approves, and offers constructive advice at the initial crucial research proposal stage; and the postgraduate student who is learning how to conduct quality research.

We recommend that, on account of its ever wider application, MMR training in particular be intensified at the early stages of postgraduate study to ensure a strong and sound conceptualisation of the research design. Training should also be strengthened for all those with supervisory and oversight roles, to enhance their expertise and enable them to provide the support that students and study leaders require. At all levels, clear understanding of MMR and what it involves at the early stages of a research project will help to drive postgraduate success in studies that benefit from the employ of this novel, important and increasingly preferred approach.

\section{ACKNOWLEDGEMENTS}

The author wishes to acknowledge the contributions made in the data collection process of this study by Tukiso Moloantoa, Rudzani Mhlari - Research Development Grant Officers; Kgabo Selolo, Research Assistant; and Treasure Malatjie, DST-NRF Research Intern. 


\section{REFERENCES}

Bamberger, M. 2012. Introduction to mixed methods in impact evaluation. Impact Evaluation Notes 3: $1-38$.

Bazeley, P. 2003. Defining ‘early career’ in research. Higher Education 45(3): 257-279.

Bazeley, P. 2004. Issues in mixing qualitative and quantitative approaches to research. In Applying qualitative methods to marketing management research, ed. R. Buber, J. Gadner and L. Richards, 141-156. London: Palgrave Macmillan.

Biesta, G. 2010. Pragmatism and the philosophical foundations of mixed methods research. Sage handbook of mixed methods in social and behavioral research 2: 95-118.

Braun, V. and V. Clarke. 2006. Using thematic analysis in psychology. Qualitative Research in Psychology 3(2): 77-101.

Bulsara, C. 1997. Using a Mixed-Method Approach to enhance and validate your research. PowerPoint presentation. https://www.nd.edu.au/downloads/research/ihrr/using_mixed_methods_approach_ to_enhance_and_validate_your_research.pdf (accessed 26 August 2016).

Burrows, T. 2013. A preliminary rubric design to Evaluate Mixed Methods research. (Unpublished doctoral dissertation). Blacksburg: Virginia Polytechnic Institute and State University.

Cameron, R. 2011. Mixed Methods Research: The five Ps framework. The Electronic Journal of Business Research Methods 9(2): 96-208. www.ejbrm.com/issue/download.html?idArticle=269 (accessed 22 March 2016).

Cameron, R. and J. F. Molina-Azorin. 2011. The acceptance of mixed methods in business and management research. International Journal of Organizational Analysis 19(3): 256-271.

Centre for Higher Education Transformation. 2010. Institutional types in higher education in South Africa. http://www.chet.org.za/files/uploads/events/dialogues_he/Session\%202a\%20Institution al\%20Types\%20in\%20SA\%20HE\%20-\%20Ian\%20Bunting.pdf (accessed 16 August 2016).

CHET see Centre for Higher Education Transformation.

Clark, V. P. and J. W. Creswell. 2011. Designing and conducting mixed methods research. $2^{\text {nd }}$ Edition. Thousand Oaks: Sage Publications.

Creswell, J. W. 2011. Controversies in mixed methods research. In The Sage handbook of qualitative research, ed. N. K. Denzin and Y. S. Lincoln, 269-284. Thousand Oaks, California: Sage Publications.

Creswell, J. W. 2013. Research design: Qualitative, quantitative, and mixed methods approaches. London: Sage Publications.

Creswell, J. W. 2014. A concise introduction to mixed methods research. New Delhi: Sage Publications.

Davies, M. B. and N. Hughes. 2014. Doing a successful research project: Using qualitative or quantitative methods. Palgrave Macmillan.

Denscombe, M. 2008. Communities of practice a research paradigm for the mixed methods approach. Journal of Mixed Methods Research 2(3): 270-283.

Easton, K. L., J. F. McComish and R. Greenberg. 2000. Avoiding common pitfalls in qualitative data collection and transcription. Qualitative Health Research 10(5): 703-707.

Gibbon, T. 2004. Creating comprehensive universities in South Africa: A concept document. Pretoria: Department of Education.

Golafshani, N. 2003. Understanding reliability and validity in qualitative research. The Qualitative Report 8(4): 597-606.

Guetterman, T. C. 2016. What distinguishes a novice from an expert mixed methods researcher? Quality \& Quantity, 1-22.

Hanson, B. P. 2006. Designing, conducting and reporting clinical research: A step by step approach. 
Injury 37(7): 583-594.

Harle, J. 2012. Africa's early career researchers need support and so do their universities. The Guardian. 28 November 2012. https://www.theguardian.com/higher-education-network/blog/2012/nov/28/ early-careers-academics-african-universities (accessed 27 July 2016).

Healy, M. and C. Perry. 2000. Comprehensive criteria to judge validity and reliability of qualitative research within the realism paradigm. Qualitative Market Research: An International Journal 3(3): 118-126.

Hurmerinta-Peltomäki, L. and N. Nummela. 2006. Mixed methods in international business research: A value-added perspective. Management International Review 46(4): 439-459.

Johnson, B. and L. Christensen. 2008. Educational research: Quantitative, qualitative, and mixed approaches. California: Sage Publications.

Kawulich, B., M. Garner and C. Wagner. 2009. Students' conceptions - and misconceptions - of social research. Qualitative Sociology Review 5(3): 5-25.

Leech, N. L. and A. J. Onwuegbuzie. 2009. A typology of mixed methods research designs. Quality \& Quantity 43(2): 265-275.

Leech, N. L., A. B. Dellinger, K. B. Brannagan and H. Tanaka. 2010. Evaluating mixed research studies: A mixed methods approach. Journal of Mixed Methods Research 4(1): 17-31.

Long, A. F., M. Godfrey, T. Randall, A. Brettle and M. J. Grant. 2002. HCPRDU Evaluation tool for mixed methods studies. University of Leeds, Nuffield Institute for Health, Leeds. http://tinyurl. com/14jaaj3 (accessed 24 May 2016).

MacGregor, K. 2010. South Africa: New university clusters emerge. University World News 23 May 2010. (Issue 125). http://www.universityworldnews.com/article.php?story=201005231041197 (accessed 13 November 2016).

Mann, C. J. 2003. Observational research methods. Research design II: Cohort, cross sectional, and case-control studies. Emergency Medicine Journal 20(1): 54-60.

McMillan, J. H. and S. Schumacher. 2014. Research in education: Evidence-based inquiry. Indiana: Prentice Hall.

Mertens, D. M. 2014. Research and evaluation in education and psychology: Integrating diversity with quantitative, qualitative, and mixed methods. London: Sage Publications.

Meyer, J., M. Shanahanb and R. Laugkschc. 2005. Students' conceptions of research. I: A qualitative and quantitative analysis. Scandinavian Journal of Educational Research 49(3): 225-244.

Munhall, P. L. 2012. Nursing research. London: Jones \& Bartlett Learning.

Munyaradzi-Muchacha, B. S. W. and E. Mtetwa. 2015. Social and economic barriers to exclusive breastfeeding in rural Zimbabwe. International Journal of MCH and AIDS (IJMA) 3(1): 16-21.

Murtonen, M. and E. Lehtine. 2005. Conceptions of research and methodological learning. Scandanavian Journal of Educational Research 49(3): 217-224.

National Planning Commission. 2012. National Development Plan 2030: Our future, make it work. http://www.gov.za/documents/national-development-plan-2030-our-future-make-it-work (accessed 27 September 2016).

Ngulube, P. and B. Ngulube. 2015. Mixed methods research in The South African Journal of Economic and Management Sciences: An investigation of trends in the literature. South African Journal of Economic and Management Sciences 18(1): 1-13.

NPC see National Planning Commission.

O'Cathain, A. 2009. Editorial: Mixed methods research in the health sciences: A quiet revolution. Journal of Mixed Methods Research 3(1): 3-6.

O’Cathain, A., E. Murphy and J. Nicholl. 2010. Three techniques for integrating data in mixed methods studies. BMJ 341: c4587.

Odom, S. L., E. Brantlinger, R. Gersten, R. H. Horner, B. Thompson and K. R. Harris. 2005. Research 
in special education: Scientific methods and evidence-based practices. Exceptional Children 71(2): 137-148.

Onwuegbuzie, A. J. 2002. Why can't we all get along? Towards a framework for unifying research paradigms. Education 122(3): 518.

Onwuegbuzie, A. J., R. B. Johnson and K. M. Collins. 2011. Assessing legitimation in mixed research: A new framework. Quality \& Quantity 45(6): 1253-1271.

Plowright, D. 2013. To what extent do postgraduate students understand the principles of mixed methods in educational research? International Journal of Multiple Research Approaches 7(1): 66-82.

Ponce, O. A. and Pagán-Maldonado, N. 2015. Mixed methods research in education: Capturing the complexity of the profession. International Journal of Educational Excellence 1(1): 111-135.

Ritchie, J., J. Lewis, C. M. Nicholls and R. Ormston. (Eds.). 2003. Qualitative research practice: A guide for social science students and researchers. California: Sage Publications.

Sandelowski, M. 2004. Using qualitative research. Qualitative Health Research 14(10): 1366-1386.

Seale, C. 1999. Quality in qualitative research. Qualitative inquiry 5(4): 465-478.

Starr, M. 2012. Qualitative and Mixed-Methods Research in Economics: Surprising growth, promising future. Journal of Economic Surveys 28(2): 238-264.

Stockman, C. 2015. Achieving a Doctorate through Mixed Methods Research. The Electronic Journal of Business Research Methods 13(2): 74-84.

Stubba, J., K. Pyhältöb and K. Lonkaac. 2014. Conceptions of research: The doctoral student experience in three domains. Studies in Higher Education 39(2): 251-264.

Tashakkori, A. and C. Teddlie. 2008. Quality of inferences in mixed methods research: Calling for an integrative framework. Advances in Mixed Methods Research: 101-119.

Tashakkori, A. and C. Teddlie. 2010a. Epilogue: current developments and emerging trends in integrated research methodology. In Sage handbook of Mixed Methods in Social and behavioral research, ed. A. Tashakkori and C. Teddlie, 803-826. New Delhi: Sage Publications.

Tashakkori, A. and C. Teddlie. (Eds.). 2010b. Sage handbook of mixed methods in social and behavioral research. New Delhi: Sage Publications.

Teddlie, C. and A. Tashakkori. 2011. Mixed methods research. The Sage handbook of qualitative research. New Delhi: Sage Publications.

Ukwuoma, U. 2015. Mixed Research: Exploring postgraduate students' perspectives. International Journal of Novel Research in Education and Learning 2(4): 134-141.

Vermunt, J. 2005. Conceptions of Research and Methodology Learning: A commentary on the Special Issue. Scandinavian Journal of Educational Research 49(3): 329-334.

Wong, P. T. P. 2016. How to write a research proposal. Langley: Trinity Western University Langley. http://www.meaning.ca/archives/archive/art_how_to_write_P_Wong.htm (accessed 26 July 2016). 